\title{
Le progrès en néphrologie: béné- fique pour les patients et les coûts
}

Pierre-Yves Martin

Service de Néphrologie HUG, Président Société Suisse de Néphrologie

La diminution de la progression des maladies rénales vers l'insuffisance rénale terminale: peu visible mais bien présente, elle représente un bénéfice pour les patients et une économie financière sur le long terme. Tout patient qui ne progresse pas vers la dialyse bénéficie d'une qualité de vie plus élevée - et représente une économie d'environ 250000 CHF.

La dialyse et la transplantation rénale disponibles en Suisse depuis les années 70 permettent à des milliers de personnes de vivre malgré une fonction rénale insuffisante à la vie. Depuis les années 70, le recours à ces traitements de substitution rénale n'a cessé d'augmenter en Suisse et actuellement plus de 10000 personnes vivent en Suisse grâce à ces traitements [1] Ceux-ci permettent une survie conséquente puisque $55 \%$ des personnes entre 18 et 64 ans ayant nécessité un de ces traitements en 2004 sont encore en vie 10 ans plus tard.

Mme $X$ a une maladie rénale congénitale qui évolue vers l'insuffisance terminale à 30 ans. Elle débute la dialyse péritonéale ambulatoire qui lui permet de continuer à travailler à $100 \%$ comme expert comptable et elle est mise en liste d'attente pour une transplantation rénale. Son mari désire lui donner un rein de son vivant mais ils ne sont pas du même groupe sanguin. Après 2 ans de dialyse péritonéale, la patiente commence à être plus fatiguée et a de la peine à maintenir son travail. Le couple prend alors I'option d'une transplantation $A B O$ incompatible (ils ont des différents groupes sanguins) qui est possible depuis plusieurs années en Suisse. La transplantation est un succès et $\mathrm{Mme} X$ reprend son travail à 100\% 6 mois après la transplantation. Deux ans après, le couple a son premier enfant.

Les techniques de dialyse se sont améliorées et diversifiées notamment avec l'avènement de la dialyse péritonéale ambulatoire et plus récemment de l'hémodialyse à domicile entraînant une nette amélioration de la qualité de vie. La transplantation rénale est le meilleur traitement de l'insuffisance rénale terminale et les progrès réalisés ont rendu la greffe plus accessible et mieux tolérée. Le don vivant s'est beaucoup développé et on peut maintenant faire des greffes de reins $\mathrm{ABO}$ incompatibles et un programme de dons croisés permet d'améliorer les chances de trouver des donneurs compatibles mais il y a des restrictions. La première est d'ordre médical car plus de 50\% des patients qui arrivent en insuffisance rénale terminale ne peuvent pas bénéficier de ce traitement, par ex. à cause des facteurs de risques cardiovasculaires, ou en raison de leur âge avancé. La deuxième est liée au faible taux de transplantation à partir de donneurs décédés en Suisse qui n'est que partiellement compensé par un taux élevé de transplantation à partir d'un donneur vivant, en règle générale un conjoint ou un membre de la famille. Ceci entraîne un nombre croissant de personnes en liste d'attente et un temps d'attente moyen très long (entre 3 et 4 ans). Ceci a également des consé-

Les coûts augmentent chaque année - parce que le nombre de patients et l'espérance de vie par patient croissent.

quences financières car la transplantation rénale est le traitement le moins coûteux de l'insuffisance rénale terminale.

Néanmoins, ces traitements restent très lourds dans un système de santé en raison des infrastructures nécessaires pour pratiquer la dialyse ou la transplantation rénale et les coûts que cela génère. Comme le nombre de patients et l'espérance de vie par patient croissent, les coûts augmentent chaque année. C'est pour cette raison que le traitement de l'insuffisance rénale terminale est la cible des décideurs en santé et que des économies sont demandées dans ces domaines. Ceci entraîne de grosses tensions entre la communauté néphrologique et les organismes payeurs car la 
majorité des frais liés à des structures est difficilement compressible.

\section{Le défi: diminuer le nombre de patients arrivant en insuffisance rénale terminale.}

La meilleure façon d'améliorer la situation des patients et de réduire les coûts de traitement est une diminution du nombre de patients arrivant en insuffisance rénale terminale (IRT). Ce défi parait difficile à relever avec le vieillissement de la population sachant que ce sont les patients de plus de 75 ans qui forment le plus gros groupe de patients en IRT. D'autre part, les deux causes les plus fréquentes d'insuffisance rénale terminale, soit le diabète de type 2 et l'hypertension artérielle, ne cessent d'augmenter [2].

Monsieur $Y$, âgé de 65 ans, fumeur, souffre d'un diabète de type 2 et d'une hypertension depuis 20 ans, accompagnés d'une prise de poids de plus de $20 \mathrm{~kg}$. II n'a jamais pu suivre un régime en raison d'une grosse activité professionnelle et sociale et les traitements de son diabète et de son hypertension sont insatisfaisants. II développe une insuffisance rénale progressive et on lui prédit la dialyse dans les 3 ans. Débutant sa retraite, il s'astreint alors à un régime adapté, modéré en sel et en protéines, riches en légumes et il arrête de fumer et reprend une activité physique adaptée ( $3 \times 1$ heure de marche/semaine). Un traitement médicamenteux permet d'améliorer ses valeurs tensionnelles et de corriger l'acidose métabolique qui accompagne son insuffisance rénale. A 67 ans, il a perdu $13 \mathrm{~kg}$, le diabète est mieux contrôlé et son insuffisance rénale s'est stabilisée. Si la perspective de la dialyse n'est pas totalement exclue, elle s'éloigne à 15 ans ou plus.

Néanmoins, nous assistons depuis deux décennies à de gros progrès dans la compréhension de la pathogénèse de l'insuffisance rénale chronique. La maladie rénale chronique peut survenir dans différents contextes: elle peut être la conséquence d'une atteinte rénale aiguë (insuffisance rénale aigue de toutes origines) qui entraîne des lésions rénales irréversibles. Elle peut être aussi la conséquence de maladies systémiques autoimmunes ou métaboliques dont les traitements spécifiques ne permettent pas d'éviter des lésions rénales. Dans les deux situations, ceci mène à une perte de néphrons, qui est l'unité structurelle et fonctionnelle du rein. Cette perte entraîne une surcharge des néphrons encore fonctionnels accélérant le processus de vieillissement qui mènent à la dégénérescence progressive des néphrons. Ceci aggrave encore la perte de néphrons et le processus se poursuit progressivement jusqu'à la perte totale de fonction.

La compréhension de ce phénomène a mené au concept de "néphroprotection" qui correspond à un ensemble de mesures médicamenteuses, diététiques et d'hy- giène de vie. Nous n'arrivons pas encore à guérir une insuffisance rénale chronique mais nous arrivons à freiner voire stopper sa progression. Pour cela, nous avons plusieurs outils que nous pouvons combiner. Des médicaments spécifiques (IEC/sartans) sont incon-

Nous n'arrivons pas encore à guérir une insuffisance rénale chronique mais nous arrivons à freiner voire stopper sa progression.

tournables et leur utilité est bien démontrée [3]. Dans toutes les études publiées, ces traitements freinent la progression de l'insuffisance rénale, néanmoins avec une efficacité variable. L'abstention du tabac, la diminution de la charge acide par des régimes limités en protéines et par l'addition d'aliments alcalins s'avèrent également efficaces. L'utilisation précoce de statines a un effet protecteur cardiovasculaire. L'avènement des inhibiteurs SGLT2 dans le traitement du diabète de type 2 est très prometteur dans la néphropathie diabétique et les premières études démontrent qu'ils ralentissent la progression de la néphropathie diabétique [4].

\section{Ralentir la progression de l'insuffisance rénale avec des thérapies médicamen- teuses}

M. Z, âgé de 40 ans, constate que ses jambes enflent énormément en quelques jours. On diagnostique une glomérulonéphrite membraneuse associée à des anticorps anti PLA2R, une maladie auto-immune qui est la cause principale des glomérulonéphrites membraneuses primaires. Le risque d'évoluer vers l'insuffisance rénale terminale est de $50 \%$ sans traitement et les traitements disponibles associaient des hautes doses de cortisone et de l'Endoxan, un traitement immunosuppresseur qui est associé avec beaucoup d'effets secondaires. On choisira de lui donner le rituximab, un traitement non encore enregistré dans cette indication, mais pour lequel plusieurs études ont démontré son efficacité et son meilleur profil d'effets secondaires. II entrera en rémission 6 mois après le traitement et 5 ans après, il n'a pas présenté de récidive et sa fonction rénale est normale.

Parmi les causes d'insuffisance rénale, il y a aussi une affection nommée glomérulonéphrite. Comme la grande majorité des patients concernés évolue vers l'insuffisance rénale terminale (IRT), cette maladie représente une cause importante d'IRT. Les traitements biologiques comme le rituximab ou l'eculizumab, développés pour des indications non néphrologiques, s'avèrent être très efficaces dans le traitement de nombreuses glomérulonéphrites [5]. Encore peu utilisés dans le monde en raison de leurs prix pour ces indications qui ne sont pour la plupart pas enregistrées, ils devraient cependant mener à une amélioration du pro- 
nostic rénal des glomérulonéphrites. Nous le constatons déjà en Suisse et aux USA.

Il y a également bon espoir dans le traitement de la maladie polykystique rénale, une maladie héréditaire qui entraîne la formation de nombreux kystes dans les reins. Depuis une année, un médicament spécifique, le tolvaptan, est disponible [6]. Il freine la croissance des kystes et ralentit en conséquence la progression de l'insuffisance rénale avec une efficacité équivalente à celle des IEC sur les premières années. Sachant que $50 \%$ des personnes souffrant de cette maladie héréditaire atteignent l'insuffisance rénale terminale, représentant plus de $10 \%$ des patients, l'impact devrait se ressentir dans quelques années si ce traitement confirme son efficacité sur le long terme.

\section{En plus du bénéfice pour les patients, des traitements efficaces sont économi- quement intéressants}

Au-delà du bénéfice pour les patients, les traitements qui peuvent éviter la progression vers l'insuffisance rénale terminale sont le meilleur moyen de diminuer le coût financier de l'insuffisance rénale. Les Américains ont estimé que tout patient qui ne progresse pas vers la dialyse représente une économie de 250000 \$ [7]. En Suisse, cette économie devrait être équivalente voire même plus importante, soit de l'ordre de $250000 \mathrm{CHF}$. En 2015, nous avons mis 848 personnes en dialyse. Une diminution de $10 \%$ de l'incidence de mise en dialyse représenterait en potentiel de 21 millions de francs d'économie pour la santé suisse. Les médicaments disponibles depuis peu et les médicaments en développement ont largement ce potentiel.

\section{Résumé}

Depuis les années 70, les techniques de dialyse et la transplantation se sont perfectionnées et diversifiées élargissant les possibilités de prise en charge et améliorant la survie et la qualité de vie. Le recours à ces traitements n'a cessé d'augmenter, et actuellement 10000 personnes vivent en Suisse grâce à ces traitements.

\section{Les traitements qui peuvent éviter la progres-} sion vers l'insuffisance rénale terminale sont le meilleur moyen de diminuer le coût financier de l'insuffisance rénale.

Néanmoins, ces traitements absorbent des ressources significatives de notre système de santé en raison des infrastructures nécessaires et des coûts que cela génère. Au-delà du bénéfice pour les patients, les traitements qui peuvent éviter la progression vers l'insuffisance rénale terminale sont le meilleur moyen de diminuer le coût financier de l'insuffisance rénale. Tout patient qui ne progresse pas vers la dialyse représente une économie d'environ $250000 \mathrm{CHF}$. Vu le nombre de patients concernés, les médicaments disponibles depuis peu et les médicaments en développement offrent un grand potentiel pour le traitement - et pour l'économie.

\section{Références}

1 Pippias M, Kramer A, Noordzij M, et al. The European Renal Association - European Dialysis and Transplant Association Registry Annual Report 2014: a summary. Clin Kidney J.2017 Apr;10(2):154-69.

2 Heaf J. Current trends in European renal epidemiology. Clin Kidney J.2017 Apr;10(2):149-53.

3 Ruiz-Hurtado G, Sarafidis P, Fernández-Alfonso MS, et al. Global cardiovascular protection in chronic kidney disease. Nat Rev Cardiol. 20160ct;13(10):603-8.

4 Wanner C, Inzucchi SE, Lachin JM,et al. EMPA-REG OUTCOME Investigators. Empagliflozin and Progression of Kidney Disease in Type 2 Diabetes. N Engl J Med.2016 Jul 28;375(4):323-34.

5 Holdsworth SR, Gan PY, Kitching AR. Biologics for the treatment of autoimmune renal diseases. Nat Rev Nephrol. 2016 Apr;12(4):217-31.

6 Gansevoort RT, Arici M, Benzing T, et al. Recommendations for the use of tolvaptan in autosomal dominant polycystic kidney disease: a position statement on behalf of the ERA-EDTA Working Groups on Inherited Kidney Disorders and European Renal Best Practice. Nephrol Dial Transplant. 2016 Mar;31(3):337-48.

7 Lederer ED. America's kidney disease epidemic merits congressional attention. The Hill. Thehill.com/blogs/congressblog/healthcare/ 